\title{
On certain generalized difference sequence spaces with some of their topological properties defined by a sequence of moduli
}

\author{
Zakawat U. Siddiqui, Abayomi O. Mosaku \\ Department of Mathematics and Statistics, University of Maiduguri, Nigeria
}

\begin{abstract}
The idea of difference sequences $X(\Delta)=\left\{x=\left(x_{k}\right): \Delta x \in X\right\}$ with $X=l_{\infty}, c$ and $c_{0}$ was introduced by kizmaz. In this paper, using the sequence of moduli we define some generalized sequence spaces and give various topological properties of these spaces with some of their inclusion relations. Furthermore, we study some of their properties, such as solidity, symmetricity, convergence free and so on.
\end{abstract}

Keywords: Difference sequence space, Paranorm, Seminorm, Sequence of moduli

\section{Introduction}

Let $\omega$ be the set of all sequences of real or complex numbers (scalar). It is not difficult to see that any arbitrary sequence space $X$ can be shown to be a vector space by defining its vector addition and multiplication as follows:

Let

$$
x=\left(x_{1}, x_{2}, x_{3}, . .\right), y=\left(y_{1}, y_{2}, y_{3}, . .\right)
$$

be two arbitrary elements of $X$ i.e $x, y \in X$ and $\alpha \in K$ then

$$
x+y=\left(x_{1}+y_{1}, x_{2}+y_{2}, \ldots\right) \in X
$$

$$
\begin{aligned}
\alpha x & =\alpha\left(x_{1}, x_{2}, x_{3}, \ldots\right) \\
& =\left(\alpha x_{1}, \alpha x_{2}, \alpha x_{3}, \ldots\right) \in X
\end{aligned}
$$

In view of this, a sequence space is any linear subspace of $\omega$. The spaces $l_{\infty}, c$ and $c_{0}$ are of particular interest in this research work.

In 1981, Kizmaz [1] introduces the notion of difference sequence spaces as follows:

$$
\begin{gathered}
X(\Delta)=\left\{x=\left(x_{k}\right) \in \omega:\left(\Delta x_{k}\right) \in X\right\} \\
X=l_{\infty}, c \text { and } c_{0} X(\Delta)=\left\{x=\left(x_{k}\right) \in \omega:\left(\Delta x_{k}\right) \in X\right\}
\end{gathered}
$$

where

They are Banach spaces with the norm

$$
\Delta x=\Delta x_{k}=\left(x_{k}-x_{k+1}\right)
$$

$$
\square x \square_{\Delta}=\left|x_{1}\right|+\square \Delta_{x} \square_{\infty} .
$$

Later on, this notion was generalized in the following way:

$$
X\left(\Delta^{m}\right)=\left\{x=\left(x_{k}\right) \in \omega:\left(\Delta^{m} x_{k}\right) \in X\right\}
$$

for $X=l_{\infty}, c$ and $c_{0}$, where $m \in \square$, where

$$
\begin{aligned}
& \Delta^{m} x=\Delta^{m} x_{k}=\left(\Delta^{m-1} x_{k}-\Delta^{m-1} x_{k+1}\right) \\
& \Delta x_{k}=\left(x_{k}-x_{k+1}\right) \\
& \text { and } \Delta^{0} x=\left(x_{k}\right) .
\end{aligned}
$$

such that

$$
\Delta^{m} x_{k}=\sum_{i=0}^{m}(-1)^{i}\left(\begin{array}{c}
m \\
i
\end{array}\right) x_{k+i}
$$

They are also Banach spaces with norm defined by

$$
\square x \square_{\Delta}=\sum_{i=1}^{m}\left|x_{i}\right|+\square \Delta^{m} x \square_{\infty}
$$

(Et and Colak, [2]) 
Worthy it is to mention that recently, the sequence spaces $X\left(\Delta^{m}\right)$ were generalized by Et and Esi [3] to the following sequence spaces

$$
X\left(\Delta_{v}^{m}\right)=\left\{x=\left(x_{k}\right) \in \omega:\left(\Delta_{v}^{m} x_{k}\right) \in X\right\}
$$

for $X=l_{\infty}, c$ and $c_{0}$, where

$$
\Delta_{v}^{0} x=\left(v_{k} x_{k}\right), \Delta_{v} x_{k}=\left(v_{k} x_{k}-v_{k+1} x_{k+1}\right)
$$

and

such that

$$
\Delta_{v}^{m} x=\Delta_{v}^{m} x_{k}=\left(\Delta_{v}^{m-1} x_{k}-\Delta_{v}^{m-1} x_{k+1}\right)
$$

$$
\Delta_{v}^{m} x_{k}=\sum_{i=0}^{m}(-1)^{i}\left(\begin{array}{c}
m \\
i
\end{array}\right) v_{k+1} x_{k+i}
$$

and $v=\left(v_{k}\right) \neq 0$ is any fixed sequence of non-zero numbers for all $k \in \square$. (Et and Esi,[3])

Subsequently, difference sequence spaces have been studied by Asma and Et [4], Bektas et al [5], Colak [6], Isik [7] and Mursaleen [8].

Definition (See Nakano [9]): A function $f:[0, \infty) \rightarrow[0, \infty)$ is called a modulus if

i.) $\quad f(t)=0$ if and only if $t=0$

ii.) $\quad f(t+u) \leq f(t)+f(u)$ for all $t, u \geq 0$

iii.) $\quad f$ is increasing, and

iv.) $\quad f$ is continuous from the right of 0 .

Let $X$ be a sequence space. Then the sequence space $X(f)$ is defined by

$$
X(f)=\left\{x=\left(x_{k}\right) \in \omega:\left(f\left(\left|x_{k}\right|\right)\right) \in X\right\} .
$$

Kolk [10] gave an extension of $X(f)$ by considering a sequence of moduli $F=\left(f_{k}\right)$ i.e

$$
X(F)=\left\{x=\left(x_{k}\right)_{k=1}^{\infty} \in \omega:\left(f_{k}\left(\left|x_{k}\right|\right)\right) \in X\right\},
$$

where $X=l_{\infty}, c$ or $c_{0}$.

Later, Gaur and Mursaleen [11] defined the following sequence spaces

$$
\begin{aligned}
& l_{\infty}(F, \Delta)=\left\{x=\left(x_{k}\right) \in \omega:\left(x_{k}\right) \in l_{\infty}(F)\right\} \\
& c_{0}(F, \Delta)=\left\{x=\left(x_{k}\right) \in \omega:\left(x_{k}\right) \in c_{0}(F)\right\}
\end{aligned}
$$

Recently Khan [12] defined the following sequence spaces

$$
X(F, p)=\left\{x=\left(x_{k}\right)_{k=1}^{\infty} \in \omega:\left(f_{k}\left(\left|x_{k}\right|\right)\right) \in X(p)\right\}
$$

In particular,

$$
\begin{gathered}
l_{\infty}(F, p)=\left\{x=\left(x_{k}\right)_{k=1}^{\infty} \in \omega: \sup _{k} f_{k}\left|x_{k}\right|^{p_{k}}<\infty\right\}, \\
c_{0}(F, p)=\left\{x=\left(x_{k}\right)_{k=1}^{\infty} \in \omega: f_{k}\left(\left|x_{k}\right|^{p_{k}}\right) \rightarrow 0 ;(k \rightarrow \infty)\right\}, \\
l_{\infty}\left(F, p, \Delta^{m}\right)=\left\{x=\left(x_{k}\right)_{k=1}^{\infty} \in \omega:\left(\Delta^{m} x_{k}\right) \in l_{\infty}(F, p)\right\}, \\
c_{0}\left(F, p, \Delta^{m}\right)=\left\{x=\left(x_{k}\right)_{k=1}^{\infty} \in \omega:\left(\Delta^{m} x_{k}\right) \in c_{0}(F, p)\right\} .
\end{gathered}
$$

For any sequence of moduli $F=\left(f_{k}\right)$

Khan [12] used the following lemmas to prove few results on the inclusion of the above spaces.

Lemma 1: The condition $\sup _{k} f_{k}(t)<\infty, t>0$ holds if and only if there exist a point $t_{0}>0$ such that $\sup _{k} f_{k}\left(t_{0}\right)<\infty$. 
Lemma 2: The condition $\underset{k}{\inf } f_{k}(t)>0, t>0$ holds if and only if there exist a point $t_{0}>0$ such that ${ }_{k}^{\text {inf }} f_{k}\left(t_{0}\right)>0$.

A modulus may be bounded or unbounded. Maddox [13]) and Ruckle [14] used a modulus function to construct some sequence spaces.

Later on, modulus function was investigate by Bhardwaj [15], Bilgin 16], Connor [17], Esi [18] and many others.

Let $P=\left(p_{k}\right)$ be a sequence of strictly positive real numbers and $s \geq 0$. Let $X$ be a seminormed space over the field $\square$ of complex numbers with the seminorm q. The symbol $\omega(X)$ denotes the space of all sequences defined over $X$. Let $V=\left(v_{k}\right)$ be any fixed sequences of non-zero complex numbers. We define the following sequence spaces as follows.

$$
\begin{gathered}
c\left(\Delta_{v}^{m}, f, p, q, s\right)=\left\{x=\left(x_{k}\right) \in \omega: \lim _{k \rightarrow \infty} k^{-s}\left[f\left(q\left(\Delta_{v}^{m} x_{k}-l\right)\right)\right]^{p_{k}}=0, l \in X\right\} \\
c_{0}\left(\Delta_{v}^{m}, f, p, q, s\right)=\left\{x=\left(x_{k}\right)_{k=0}^{\infty} \in \omega: \lim _{k \rightarrow \infty} k^{-s}\left[f\left(q\left(\Delta_{v}^{m} x_{k}\right)\right)\right]^{p_{k}}=0,\right\} \\
l_{\infty}\left(\Delta_{v}^{m}, f, p, q, s\right)=\left\{x=\left(x_{k}\right)_{k=0}^{\infty} \in \omega: \sup _{k} k^{-s}\left[f\left(q\left(\Delta_{v}^{m} x_{k}\right)\right)\right]^{p_{k}}<\infty\right\},
\end{gathered}
$$

where $f$ is a modulus function.

The following inequality will be used throughout this research.

Let $P=\left(p_{k}\right)$ be a sequence of positive real number with

$0<p_{k} \leq \sup _{k} p_{k}=H, D=\max \left(1,2^{H-1}\right)$. then, for $a_{k}, b_{k} \in \mathbb{C}$ we have.

$$
\left|a_{k}+b_{k}\right|^{p_{k}} \leq D\left\{\left|a_{k}\right|^{p_{k}}+\left|b_{k}\right|^{p_{k}}\right\}
$$

Some well known spaces as we shall see later, are obtained by specializing $f, s, q, v$ and $m$.

i.) If $f(x)=x, m=0, V=\left(v_{k}\right)=(1,1,1, \ldots)$ and $q(x)=|x|$, then $c\left(\Delta_{v}^{m} f, p, q, s\right)=c(p, s)$,

$c_{0}\left(\Delta_{v}^{m} f, p, q, s\right)=c_{0}(p, s)$, and $l_{\infty}\left(\Delta_{v}^{m} f, p, q, s\right)=l_{\infty}(p, s) \ldots .($ See Basarir, [19])

ii.) If $f(x)=x, m=0, V=\left(v_{k}\right)=(1,1,1, \ldots), s=0$ and $q(x)=|x|$ then

$c\left(\Delta_{v}^{m} f, p, q, s\right)=c(p), c_{0}\left(\Delta_{v}^{m} f, p, q, s\right)=c_{0}(p)$ and $l_{\infty}\left(\Delta_{v}^{m} f, p, q, s\right)=l_{\infty}(p)$ (See Maddox, [20]) If $m=0$ and $q(x)=|x|, V=\left(v_{k}\right)=(1,1,1, \ldots)$, then

$c\left(\Delta_{v}^{m} f, p, q, s\right)=c(p, f, s), c_{0}\left(\Delta_{v}^{m} f, p, q, s\right)=c_{0}(p, f, s)$ and $l_{\infty}\left(\Delta_{v}^{m} f, p, q, s\right)=l_{\infty}(p, f, s)$ (Esi, [18]).

Definition (Gulcan and Cigdem, [21]): Let $f=\left(f_{k}\right)$ be sequence of moduli $X$ be a seminormed space over the field $\square$ of complex number with the seminorm $q, p=\left(p_{k}\right)$ be a sequence of strictly positive real numbers and $u \in U$. By $\omega(X)$ we shall denote the space of all sequences defined over $X$. let $v=\left(v_{k}\right)$ be any fixed sequence of nonzero complex number. Now we define the following sequence spaces

and

$$
\begin{aligned}
& l_{\infty}\left(\Delta_{v}^{m}, F, p, q, u\right)=\left\{x \in \omega: \sup _{k} u_{k}\left[F_{k}\left(q\left(\Delta_{v}^{m} x_{k}\right)\right)\right]^{p_{k}}<\infty\right\} \\
& c\left(\Delta_{v}^{m}, F, p, q, u\right)=\left\{x \in \omega: \lim _{k \rightarrow \infty} u_{k}\left[f_{k}\left(q\left(\Delta_{v}^{m} x_{k}-l\right)\right)\right]^{p_{k}}=0\right\} \\
& c_{0}\left(\Delta_{v}^{m}, F, p, q, u\right)=\left\{x \in \omega: \lim _{k \rightarrow \infty} u_{k}\left[f_{k}\left(q\left(\Delta_{v}^{m} x_{k}\right)\right)\right]^{p_{k}}=0\right\}
\end{aligned}
$$

For $p_{k}=1$ for all $k \in \square$ we write these spaces as $l_{\infty}\left(\Delta_{v}^{m}, F, q, u\right), c\left(\Delta_{v}^{m}, F, q, u\right)$ and $c_{0}\left(\Delta_{v}^{m}, F, q, u\right)$

For $u_{k}=1$ for all $k \in \square$, we write these spaces as $l_{\infty}\left(\Delta_{v}^{m}, F, p, q\right), c\left(\Delta_{v}^{m}, F, p, q\right)$ and $c_{0}\left(\Delta_{v}^{m}, F, p, q\right)$. 
A sequence $x \in l_{\infty}$ is said to be almost convergent (Lorentz. [22]) if all Banach limits of $x$ coincide. Lorentz [22] proved that

$$
\hat{c}:=\left\{x=\left(x_{k}\right) \in \omega: \lim _{n} \frac{1}{n} \sum_{k=1}^{n} x_{k+s}, \text { uniformly in } S\right\}
$$

Maddox ([23] and [24]) has defined $x$ to be strongly almost convergent to $l$ if

$$
\lim _{n} \frac{1}{n} \sum_{k=1}^{n}\left|x_{k+s}-l\right|=0 \text {, uniformly in } S \text {, for some } l>0
$$

Let $p=\left(p_{k}\right)$ be a sequence of strictly positive real number. Nanda [25] defined.

$$
\begin{aligned}
& {[\hat{c}, p]:=\left\{x=\left(x_{k}\right) \in \omega: \lim _{n} \frac{1}{n} \sum_{k=1}^{n}\left|x_{k+s}-l\right|^{p_{k}}=0, \text { uniformly in } S, \text { for some } l>0\right\},} \\
& {[\hat{c}, p]_{0}:=\left\{x=\left(x_{k}\right) \in \omega: \lim _{n} \frac{1}{n} \sum_{k=1}^{n}\left|x_{k+s}\right|^{p_{k}}=0, \text { uniformly in } S\right\},} \\
& {[\hat{c}, p]_{\infty}:=\left\{x=\left(x_{k}\right) \in \omega: \sup _{n} \frac{1}{n} \sum_{k=1}^{n}\left|x_{k+s}-l\right|^{p_{k}}=0, \text { uniformly in } S\right\}}
\end{aligned}
$$

It was extended by Khan and Ayaz [26] that if $F=\left(f_{k}\right)$ is a sequence of moduli, $u=\left(u_{i}\right)$ be any sequence such that $u_{k} \neq 0$ for all $k$ and $p=\left(p_{k}\right)$ be any sequence space of strictly positive real number then w define the following sequence spaces:

$$
\begin{gathered}
{[\hat{c}, F, p]\left(\Delta_{u}^{m}\right):=\left\{x=\left(x_{k}\right) \in \omega: \lim _{n} \frac{1}{n} \sum_{k=1}^{n}\left[f_{k}\left(\left|u_{k} \Delta^{m} x_{k+s}-l\right|\right)\right]^{p_{k}}=0, \text { uniformly in } S\right\}, \text { for some } l>0,} \\
{[\hat{c}, F, p]_{0}\left(\Delta_{u}^{m}\right):=\left\{x=\left(x_{k}\right) \in \omega: \lim _{n} \frac{1}{n} \sum_{k=1}^{n}\left[f_{k}\left(\left|u_{k} \Delta^{m} x_{k+s}\right|\right)\right]^{p_{k}}=0, \text { uniformly in } S\right\},} \\
{[\hat{c}, F, p]_{\infty}\left(\Delta_{u}^{m}\right):=\left\{x=\left(x_{k}\right) \in \omega: \sup _{s, n} \frac{1}{n} \sum_{k=1}^{n}\left[f_{k}\left(\left|u_{k} \Delta^{m} x_{k+s}\right|\right)\right]^{p_{k}}<\infty, \text { uniformly in } S\right\}}
\end{gathered}
$$

For if $f_{k}(x)=x$ for every $k$, then $[\hat{c}, F, p]\left(\Delta_{u}^{m}\right)=[\hat{c}, p]$,

$$
[\hat{c}, F, p]_{0}\left(\Delta_{u}^{m}\right)=[\hat{c}, p]_{0} \text { and }[\hat{c}, F, p]_{\infty}\left(\Delta_{u}^{m}\right)=[\hat{c}, p]_{\infty} .
$$

And for if $p_{k}=1$ for all $K$ it yields

and,

$$
\begin{aligned}
& {[\hat{c}, F, p]\left(\Delta_{u}^{m}\right)=[\hat{c}, F]\left(\Delta_{u}^{m}\right),} \\
& {[\hat{c}, F, p]_{0}\left(\Delta_{u}^{m}\right)=[\hat{c}, F]_{0}\left(\Delta_{u}^{m}\right)} \\
& {[\hat{c}, F, p]_{\infty}\left(\Delta_{u}^{m}\right)=[\hat{c}, F]_{\infty}\left(\Delta_{u}^{m}\right) .}
\end{aligned}
$$

\section{Main Results}

Suppose that $F=\left(f_{k}\right)$ is a sequence of moduli, $p=\left(p_{k}\right)$ be any sequence of strictly positive real numbers with $q$, a seminorm and $u=\left(u_{k}\right) \neq 0$ be any sequence of all $K$. Let $v=\left(v_{k}\right)$ be any fixed sequence of nonzero complex numbers. Then we define the following sequence spaces:

$$
\begin{gathered}
{\left[\hat{c}_{\Delta_{v}^{m}}, F, p, q, u\right]_{c}:=\left\{x=\left(x_{k}\right) \in \omega(X): \lim _{n \rightarrow \infty} \frac{1}{n} \sum_{k=1}^{n} u_{k}\left[f_{k}\left(\left|q\left(\Delta_{v}^{m} x_{k+s}-l\right)\right|\right)\right]^{p_{k}}=0, \text { uniformly in } S\right\}, \text { for some } l>0,} \\
{\left[\hat{c}_{\Delta_{v}^{m}}, F, p, q, u\right]_{c_{0}}:=\left\{x=\left(x_{k}\right) \in \omega(X): \lim _{n \rightarrow \infty} \frac{1}{n} \sum_{k=1}^{n} u_{k}\left[f_{k}\left(\left|q\left(\Delta_{v}^{m} x_{k+s}\right)\right|\right)\right]^{p_{k}}=0, \text { uniformly in } S\right\}}
\end{gathered}
$$

and

$$
\left[\hat{c}_{\Delta_{v}^{m}}, F, p, q, u\right]_{l_{\infty}}:=\left\{x=\left(x_{k}\right) \in \omega(X): \sup _{s, n} \frac{1}{n} \sum_{k=1}^{n} u_{k}\left[f_{k}\left(\left|q\left(\Delta_{v}^{m} x_{k+s}\right)\right|\right)\right]^{p_{k}}<\infty, \text { uniformly in } S\right\} .
$$

If $q(x)=|x|, u_{k}=1$ then

$$
\left[\hat{c}_{\Delta_{v}^{m}}, F, p, q, u\right]_{c}=\left[\hat{c}_{\Delta_{v}^{m}}, F, p\right]_{c},
$$




$$
\left[\hat{c}_{\Delta_{v}^{m}}, F, p, q, u\right]_{c_{0}}=\left[\hat{c}_{\Delta_{v}^{m}}, F, p\right]_{c_{0}}
$$

and,

$$
\left[\hat{c}_{\Delta_{v}^{m}}, F, p, q, u\right]_{l_{\infty}}=\left[\hat{c}_{\Delta_{v}^{m}}, F, p\right]_{l_{\infty}} \quad \text { (Khan and Ayaz, [26]) }
$$

But if $m=0, v=\left(v_{k}\right)=(1,1,1, \ldots), f_{k}(x)=x$ for all $k, q(x)=|x|, u_{k}=1$ then,

$$
\begin{aligned}
& {\left[\hat{c}_{\Delta_{v}^{m}}, F, p, q, u\right]_{c}=[\hat{c}, p]_{c},} \\
& \quad\left[\hat{c}_{\Delta_{v}^{m}}, F, p, q, u\right]_{c_{0}}=[\hat{c}, p]_{c_{0}}
\end{aligned}
$$

and

$$
\left[\hat{c}_{\Delta_{v}^{m}}, F, p, q, u\right]_{l_{\infty}}=[\hat{c}, p]_{l_{\infty}}
$$

And if $m=0, v=\left(v_{k}\right)=(1,1,1, \ldots), f_{k}(x)=x, q(x)=|x|, p_{k}=1$ and $u_{k}=1$ then

$$
\begin{aligned}
& \left.\qquad \hat{c}_{\Delta_{v}^{m}}, F, p, q, u\right]_{c}=[\hat{c}]_{c}, \\
& \text { and } \quad\left[\hat{c}_{\Delta_{v}^{m}}, F, p, q, u\right]_{c_{0}}=[\hat{c}]_{c_{0}} \\
& {\left[\hat{c}_{\Delta_{v}^{m}}, F, p, q, u\right]_{l_{\infty}}=[\hat{c}]_{l_{\infty}} \text { (See Maddox [23] and [24]) }}
\end{aligned}
$$

If $F=\left(f_{k}\right)$ be a sequence of moduli then $\left[\hat{c}_{\Delta_{v}^{m}}, F, p, q, u\right]_{c_{0}} \subset\left[\hat{c}_{\Delta_{v}^{m}}, F, p, q, u\right]_{c} \subset\left[\hat{c}_{\Delta_{v}^{m}}, F, p, q, u\right]_{l_{\infty}}$ are strict.

Proof. The first inclusion is clear. We shall only establish the second inclusion.

Suppose that $x \in\left[\hat{c}_{\Delta_{v}^{m}}, F, p, q, u\right]_{c}$, by the definition of modulus function and lemma1, we obtain;

$$
\begin{aligned}
& \frac{1}{n} \sum_{k=1}^{n} u_{k}\left[f_{k}\left(\left|q\left(\Delta_{v}^{m} x_{k+s}\right)\right|\right)\right]^{p_{k}} \leq D \frac{1}{n} \sum_{k=1}^{n} u_{k}\left[f_{k}\left(\left|q\left(\Delta_{v}^{m} x_{k+s}-l+l\right)\right|\right)\right]^{p_{k}} \\
& \Rightarrow \frac{1}{n} \sum_{k=1}^{n} u_{k}\left[f_{k}\left(\left|q\left(\Delta_{v}^{m} x_{k+s}\right)\right|\right)\right]^{p_{k}} \leq D \frac{1}{n} \sum_{k=1}^{n} u_{k}\left[f_{k}\left(\left|q\left(\Delta_{v}^{m} x_{k+s}-l\right)\right|\right)\right]^{p_{k}}+D \frac{1}{n} \sum_{k=1}^{n} u_{k}\left[f_{k}(|q(l)|)\right]^{p_{k}}
\end{aligned}
$$

We therefore now may choose an integer $D$ such that $q(l) \leq k$. Hence, we have

$$
\frac{1}{n} \sum_{k=1}^{n} u_{k}\left[f_{k}\left(\left|q\left(\Delta_{v}^{m} x_{k+s}\right)\right|\right)\right]^{p_{k}} \leq D \frac{1}{n} \sum_{k=1}^{n} u_{k}\left[f_{k}\left(\left|q\left(\Delta_{v}^{m} x_{k+s}-l\right)\right|\right)\right]^{p_{k}}+\max \cdot\left[1,\left(\left(k_{l}\right) f_{k}(1)\right)^{H}\right]
$$

Hence $x \in\left[\hat{c}_{\Delta_{v}^{m}}, F, p, q, u\right]_{l_{\infty}}$

To show the inclusion are strict consider the following example:

Suppose $f_{k}(x)=x, p_{k}=1, v_{k}=1, u_{k}=1$ for all $k \in \square$ and $q(x)=x$. Then, the sequence $x=\left(k^{m}\right)$ belongs to $\left[\hat{c}_{\Delta_{v}^{m}}, F, p, q, u\right]_{c}$ but does not belong to $\left[\hat{c}_{\Delta_{v}^{m}}, F, p, q, u\right]_{c_{0}}$ and sequence $x=\left((-1)^{k}\right)$ belongs to $\left[\hat{c}_{\Delta_{v}^{m}}, F, p, q, u\right]_{l_{\infty}}$ but does not belong to $\left[\hat{c}_{\Delta_{v}^{m}}, F, p, q, u\right]_{c}$. Therefore the inclusions are strict.

Theorem 2.2: Let the sequence $\left(p_{k}\right)$ be bounded. Then $\left[\hat{c}_{\Delta_{v}^{m}}, F, p, q, u\right]_{c},\left[\hat{c}_{\Delta_{v}^{m}}, F, p, q, u\right]_{c_{0}}$ and $\left[\hat{c}_{\Delta_{v}^{m}}, F, p, q, u\right]_{l_{l_{\infty}}}$ are linear spaces over the complex field $\square$.

\section{Proof:}

We shall give the proof for $\left[\hat{c}_{\Delta_{v}^{m}}, F, p, q, u\right]_{c_{0}}$ only, the others can be treated similarly.

Let $x, y \in\left[\hat{c}_{\Delta_{v}^{m}}, F, p, q, u\right]_{c_{0}}$. For $\lambda, \alpha \in \square$, there exit positive integers $M_{\lambda}$ and $N_{\alpha}$, such that $|\lambda| \leq M_{\lambda}$ and $|\alpha| \leq N_{\alpha}$. Since $f$ is subadditive, $q$ is a seminorm, and $\Delta_{v}^{m}$ is 
$\frac{1}{n} \sum_{k=1}^{n} u_{k}\left[f_{k}\left(\left|q\left(\Delta_{v}^{m}\left(\lambda x_{k+s}+\alpha x_{k+s}\right)\right)\right|\right)\right]^{p_{k}} \leq D\left(\max \left(1,\left|M_{\lambda}\right|^{H}\right)\right) \frac{1}{n} \sum_{k=1}^{n} u_{k}\left[f_{k}\left(\left|q\left(\Delta_{v}^{m} x_{k+s}\right)\right|\right)\right]^{p_{k}}$

$+D\left(\max \left(1,\left|N_{\alpha}\right|^{H}\right)\right) \frac{1}{n} \sum_{k=1}^{n} u_{k}\left[f_{k}\left(\left|q\left(\Delta_{v}^{m} x_{k+s}\right)\right|\right)\right]^{p_{k}} \rightarrow 0 k \rightarrow \infty$

Hence, $\left[\hat{c}_{\Delta_{v}^{m}}, F, p, q, u\right]_{c}$ is a linear space.

The space $\left[\hat{c}_{\Delta_{v}^{m}}, F, p, q, u\right]_{c_{0}}$ is a paranormed space, paranormed by

$g(x)=\sum_{i=1}^{m} f_{k}\left(\left|q\left(v_{i} x_{i}\right)\right|\right)+\sup _{k} \frac{1}{n} \sum_{k=1}^{n} u_{k}\left[f_{k}\left(\left|q\left(\Delta_{v}^{m} x_{k+s}\right)\right|\right)\right]^{\frac{p_{k}}{M}}$

where $M=\max \left(1, \sup _{k} p_{k}\right) ;\left[\hat{c}_{\Delta_{v}^{m}}, F, p, q, u\right]_{c}$ and $\left[\hat{c}_{\Delta_{v}^{m}}, F, p, q, u\right]_{c_{0}}$ are paranormed by $g$ if $\inf _{k} p_{k}>0$.

Proof:

Obviously, $g(x)=g(-x)$ for all $x \in\left[\hat{c}_{\Delta_{v}^{\prime \prime}}, F, p, q, u\right]_{c_{0}}$

It is trivial that $\Delta_{v}^{m} x_{k}=\bar{e}$ for $x=\bar{e}$, where $\bar{e}=(e, e, e, \ldots)$ and $e$ is the zero element of $X$.

Since $q(\bar{e})=0$ and $f(0)=0$,

We get $g(e)=0$, since $t_{k}=p_{k} / M \leq 1$,

If $a_{k}$ and $b_{k}$ are complex numbers then we have;

$$
\left|a_{k}+b_{k}\right|^{t_{k}} \leq\left|a_{k}\right|^{t_{k}}+\left|b_{k}\right|^{t_{k}}
$$

Since $M \geq 1$, the above inequality implies that

$$
\begin{aligned}
& \sum_{i=1}^{m} f_{k}\left(\left|q\left(v_{i} x_{i}+v_{i} y_{i}\right)\right|\right)+\sup _{k} \frac{1}{n} \sum_{k=1}^{n} u_{k}\left[f_{k}\left(\left|q\left(\Delta_{v}^{m}\left(x_{k+s}+y_{k+s}\right)\right)\right|\right)\right]^{\frac{p_{k}}{M}} \\
& \leq \sum_{i=1}^{m} f_{k}\left(\left|q\left(v_{i} x_{i}\right)\right|\right)+\sup _{k} \frac{1}{n} \sum_{k=1}^{n} u_{k}\left[f_{k}\left(\left|q\left(\Delta_{v}^{m} x_{k+s}\right)\right|\right)\right]^{\frac{p_{k}}{M}}+\sum_{i=1}^{m} f_{k}\left(\left|q\left(v_{i} y_{i}\right)\right|\right)+\sup _{k} \frac{1}{n} \sum_{k=1}^{n} u_{k}\left[f_{k}\left(\left|q\left(\Delta_{v}^{m} y_{k+s}\right)\right|\right)\right]^{\frac{p_{k}}{M}}
\end{aligned}
$$

Now, it follows that $\mathrm{g}$ is subadditive. Next, let $\lambda$ be a nonzero scalar. The continuity of scalar multiplication follows from the inequality.

But note that $\lambda g(x)=g(\lambda x)$

So, $g(\lambda x)=k_{\lambda} \sum_{i=1}^{m} f_{k}\left(\left|q\left(v_{i} x_{i}\right)\right|\right)+\sup _{k} \frac{1}{n} \sum_{k=1}^{n}\left(k_{\lambda}^{\frac{p_{k}}{M}}\right) u_{k}\left[f_{k}\left(\left|q\left(\Delta_{v}^{m} x_{k+s}\right)\right|\right)\right]^{\frac{p_{k}}{M}}$

$\leq \max \left(1, k_{\lambda}^{\frac{H}{M}}\right) g(x)$

where $k_{\lambda}$ is an integer such that $|\lambda|<k_{\lambda}$ and $H=\sup _{k} p_{k}$

This complete the proof of the theorem other cases will follow by applying similar technique.

Theorem 2.4: Let $F=\left(f_{k}\right)$ and $G=\left(g_{k}\right)$ be two sequences of moduli. For any two sequences $p=\left(p_{k}\right)$ and $t=\left(t_{k}\right)$ of strictly positive real seminorms $q, q_{1}$, and $q_{2}$ and $u, u_{1}$ and $u_{2} \geq 0$. Then

(i) $\left[\hat{c}_{\Delta_{v}^{m}}, F, p, q, u\right]_{z} \subseteq\left[\hat{c}_{\Delta_{v}^{m}}, G \circ F, p, q, u\right]_{z}$

$$
\left[\hat{c}_{\Delta_{v}^{m}}, F, p, q, u\right]_{z} \cap\left[\hat{c}_{\Delta_{v}^{m}}, G, p, q, u\right]_{z} \subseteq\left[\hat{c}_{\Delta_{v}^{m}}, F+G, p, q, u\right]_{z}
$$

$$
\left[\hat{c}_{\Delta_{v}^{m}}, F, p, q_{1}, u\right]_{z} \cap\left[\hat{c}_{\Delta_{v}^{m}}, F, p, q_{2}, u\right]_{z} \subseteq\left[\hat{c}_{\Delta_{v}^{m}}, F, p, q_{1}+q_{2}, u\right]_{z}
$$

\footnotetext{
If $q_{1}$ is stronger than $q_{2}$, then
} 
(v) $\left[\hat{c}_{\Delta_{v}^{m}}, F, p, q_{1}, u\right]_{z} \subseteq\left[\hat{c}_{\Delta_{v}^{m}}, F, p, q_{2}, u\right]_{z}$

(vi) If $u_{1} \leq u_{2}$, then $\left[\hat{c}_{\Delta_{v}^{m}}, F, p, q, u_{1}\right]_{z} \subseteq\left[\hat{c}_{\Delta_{v}^{m}}, F, p, q, u_{2}\right]_{z}$ where $z=l_{\infty}, c$ and $c_{0}$

Proof:

(i) We prove for $z=c$ and the rest cases will follow similarly.

Let $x \in\left[\hat{c}_{\Delta_{v}^{m}}, F, p, q, u\right]_{c}$, so that $\mu_{n}=\frac{1}{n} \sum_{k=1}^{n} u_{k}\left[f_{k}\left(\left|q\left(\Delta_{v}^{m} x_{k+s}-l\right)\right|\right)\right]^{p_{k}} \rightarrow 0,(n \rightarrow \infty)$

Let $\in>0$ and choose $\delta$ with $0<\delta<1$ such that $f(t)<\epsilon$

For $0 \leq t \leq \delta$. Now we write

$$
\begin{aligned}
& s_{1}:=\left\{k \in \square: f_{k}\left(\left|q\left(\Delta_{v}^{m} x_{k+s}-l\right)\right|\right) \leq \delta\right\}, \\
& s_{2}:=\left\{k \in \square: f_{k}\left(\left|q\left(\Delta_{v}^{m} x_{k+s}-l\right)\right|\right)>\delta\right\} .
\end{aligned}
$$

If $x \in\left[\hat{c}_{\Delta_{v}^{m}}, F, p, q, u\right]_{c}$, then for $f_{k}\left(\left|q\left(\Delta_{v}^{m} x_{k+s}-l\right)\right|\right)>\delta$,

$$
f_{k}\left(\left|q\left(\Delta_{v}^{m} x_{k+s}-l\right)\right|\right)<f_{k}\left(\left|q\left(\Delta_{v}^{m} x_{k+s}-l\right)\right|\right) \delta^{-1}<1+\left[f_{k}\left(\left|q\left(\Delta_{v}^{m} x_{k+s}-l\right)\right|\right) \delta^{-1}\right],
$$

Where $k \in s_{2}$ and $[n]$ denotes the integer part of $\mathrm{n}$.

Given $\in>0$, by definition of modulus function we have, for

$$
\begin{aligned}
& f_{k}\left(\left|q\left(\Delta_{v}^{m} x_{k+s}-l\right)\right|\right)>\delta \\
& g_{k}\left(f_{k}\left(\left|q\left(\Delta_{v}^{m} x_{k+s}-l\right)\right|\right)\right) \leq 1+\left[f_{k}\left(\left|q\left(\Delta_{v}^{m} x_{k+s}-l\right)\right|\right) \delta^{-1}\right] g_{k}(1) \\
& \quad \leq 2 g_{k}(1)\left(f_{k}\left(\left|q\left(\Delta_{v}^{m} x_{k+s}-l\right)\right|\right) \delta^{-1}\right)
\end{aligned}
$$

and hence

$$
\begin{array}{cc}
\frac{1}{n} \sum_{k=1}^{n} u_{k}\left[g_{k}\left(f_{k}\left(\left|q\left(\Delta_{v}^{m} x_{k+s}-l\right)\right|\right)\right)\right]^{p_{k}} \leq\left[2 g_{k}(1) \delta^{-1}\right]^{H} \mu_{n}<\epsilon,\left(k \in s_{1}, k>k_{2}\right) \\
\text { If } \\
x \in\left[\hat{c}_{\Delta_{v}^{m}}, F, p, q, u\right]_{c}, f_{k}\left(\left|q\left(\Delta_{v}^{m} x_{k+s}-l\right)\right|\right) \leq \delta
\end{array}
$$

Form (1) and (2) for every $k>\max \left\{k_{1}, k_{2}\right\}$,

$$
\frac{1}{n} \sum_{k=1}^{n} u_{k}\left[g_{k}\left(f_{k}\left(\left|q\left(\Delta_{v}^{m} x_{k+s}-l\right)\right|\right)\right)\right]^{p_{k}}<\epsilon
$$

Hence, $x \in\left[\hat{c}_{\Delta_{v}^{m}}, F, p, q, u\right]_{c}$

Thus,

$$
\left[\hat{c}_{\Delta_{v}^{m}}, F, p, q, u\right]_{c} \subset\left[\hat{c}_{\Delta_{v}^{m}}, F \circ G, p, q, u\right]_{c} .
$$

(ii) It follows from the following inequality

$$
\frac{1}{n} \sum_{k=1}^{n} u_{k}\left[\left(f_{k}+g_{k}\right)\left(\left|q\left(\Delta_{v}^{m} x_{k+s}-l\right)\right|\right)\right]^{p_{k}} \leq D \frac{1}{n} \sum_{k=1}^{n} u_{k}\left[f_{k}\left(\left|q\left(\Delta_{v}^{m} x_{k+s}-l\right)\right|\right)\right]^{p_{k}}+D \frac{1}{n} \sum_{k=1}^{n} u_{k}\left[g_{k}\left(\left|q\left(\Delta_{v}^{m} x_{k+s}-l\right)\right|\right)\right]^{p_{k}}
$$

(iii) It follows from the following inequality

$$
\frac{1}{n} \sum_{k=1}^{n} u_{k}\left[f_{k}\left(\left|\left(q_{1}+q_{2}\right)\left(\Delta_{v}^{m} x_{k+s}-l\right)\right|\right)\right]^{p_{k}} \leq D \frac{1}{n} \sum_{k=1}^{n} u_{k}\left[f_{k}\left(\left|q_{1}\left(\Delta_{v}^{m} x_{k+s}-l\right)\right|\right)\right]^{p_{k}}+D \frac{1}{n} \sum_{k=1}^{n} u_{k}\left[f_{k}\left(\left|q_{2}\left(\Delta_{v}^{m} x_{k+s}-l\right)\right|\right)\right]^{p_{k}}
$$

(iv.) and (v.) follows obviously.

Theorem 2.5: Let $m \geq 1$ then for all $0<i \leq m,\left[\hat{c}_{\Delta_{v}^{i}}, F, p, q, u\right]_{z} \subset\left[\hat{c}_{\Delta_{v}^{m}}, F, p, q, u\right]_{z}$ where $z=l_{\infty}, c$ or $c_{0}$.

The inclusions are strict.

Proof: We shall prove that 


$$
\left[\hat{c}_{\Delta_{v}^{i}}, F, p, q, u\right]_{l_{\infty}} \subset\left[\hat{c}_{\Delta_{v}^{m}}, F, p, q, u\right]_{l_{\infty}}
$$

For any $0<i \leq m$.

It follows from the following inequality

$\frac{1}{n} \sum_{k=1}^{n} u_{k}\left[f_{k}\left(\left|q\left(\Delta_{v}^{i} x_{k+s}\right)\right|\right)\right]^{p_{k}} \leq \frac{1}{n} \sum_{k=1}^{n} u_{k}\left[f_{k}\left(\left|q\left(\Delta_{v}^{i-1} x_{k+s}\right)\right|\right)\right]^{p_{k}}+\frac{1}{n} \sum_{k=1}^{n} u_{k}\left[f_{k}\left(\left|q\left(\Delta_{v}^{i-1} x_{k+s+1}\right)\right|\right)\right]^{p_{k}}$

That $\left(x_{k}\right) \in\left[\hat{c}_{\Delta_{v}^{i-1}}, F, p, q, u\right]_{l_{\infty}}$ implies $\left(x_{k}\right) \in\left[\hat{c}_{\Delta_{v}^{i}}, F, p, q, u\right]_{l_{x}}$

On applying the principle of induction, it follows that

$\left[\hat{c}_{\Delta_{v}^{i-1}}, F, p, q, u\right]_{l_{\infty}} \subset\left[\hat{c}_{\Delta_{v}^{m}}, F, p, q, u\right]_{l_{\infty}}$ for $i=0,1,2,3, \ldots, m-1$.

The proofs for the rest of the cases are similar.

To show that the inclusions are strict, consider the following example.

Let $X=\square, f_{k}(x)=x, p_{k}=1, v_{k}=(1,1,1, \ldots), u_{k}=1$ for all $k \in \square$ and $q(x)=|x|$.

Then for $z=c_{0}$ the sequence $\left(x_{k}\right) \notin\left[\hat{c}_{\Delta_{v}^{m}}, F, p, q, u\right]_{z}$

Under the above restrictions, consider the sequence

$x=k^{m} \in\left[\hat{c}_{\Delta_{v}^{m}}, F, p, q, u\right]_{z}$ but $x \notin\left[\hat{c}_{\Delta_{v}^{m}}, F, p, q, u\right]_{z}$ for $z=l_{\infty}$ and $c$.

Therefore the inclusions are strict.

Theorem 2.6: for any two sequences $p=\left(p_{k}\right)$ and $t=\left(t_{k}\right)$ of strictly positive real numbers and for any two seminorms $q_{1}$ and $q_{2}$ on $X$, we have

$\left[\hat{c}_{\Delta_{v}^{m}}, F, p, q_{1}, u\right]_{z} \subset\left[\hat{c}_{\Delta_{v}^{m}}, F, t, q_{2}, u\right]_{z} \neq \varnothing$ for $z=c, c_{0}$ and $l_{\infty}$.

Proof: Since the zero element belong to each of the above classes of sequences, the intersection is nonempty.

Theorem 2.7: Let $0<p_{k} \leq t_{k}$ and $\left(t_{k} / p_{k}\right)$ be bounded. Then $\left[\hat{c}_{\Delta_{v}^{m}}, F, t, q, u\right]_{z} \subset\left[\hat{c}_{\Delta_{v}^{m}}, F, p, q, u\right]_{z}$. Where $z=l_{\infty}, c$ and $c_{0}$.

Proof: We shall prove only $\left[\hat{c}_{\Delta_{v}^{m}}, F, t, q, u\right]_{c_{0}} \subset\left[\hat{c}_{\Delta_{v}^{m}}, F, p, q, u\right]_{c_{0}}$. The other inclusions can be proved similarly.

Let $x \in\left[\hat{c}_{\Delta_{v}^{m}}, F, t, q, u\right]_{c}$, write

$w_{k}=\left[f_{k}\left(\left|q\left(\Delta_{v}^{m} x_{k+s}\right)\right|\right)\right]^{t_{k}}$ and $\mu_{k}=\frac{p_{k}}{t_{k}}$, so that $0<\lambda<\lambda_{k} \leq 1$ for each $k$.

We defined the sequence $\left(r_{k}\right)$ and $\left(s_{k}\right)$ as follows:

Let $r_{k}=w_{k}$ and $s_{k}=0$ if $w<1$. Then it is clear that for all $k \in \square$, we have $w_{k}=r_{k}+s_{k}, w_{k}=r_{k}^{\lambda_{k}}+s_{k}^{\lambda_{k}}$.

Now it follows that $r_{k}^{\lambda_{k}} \leq r_{k} \leq w_{k}$ and $s_{k}^{\lambda_{k}} \leq s_{k}^{\lambda}$.

Now we are $\lim _{k} w_{k}^{\lambda_{k}} \leq \lim _{k} w_{k}+\left(\lim _{k} s_{k}\right)^{\lambda}$

This implies that $x \in\left[\hat{c}_{\Delta_{v}^{m}}, F, p, q, u\right]_{c_{0}}$ and this completes the proof.

Theorem 2.8: The sequence spaces $\left[\hat{c}_{\Delta_{v}^{m}}, F, p, q, u\right]_{z}$ for $z=l_{\infty}, c$ and $c_{0}$ are not solid for $m>0$.

Proof: Let $X=\square, f_{k}(x)=x, p_{k}=1, u_{k}=1, v_{k}=1$ for all $k \in \square$ and $q(x)=|x|$. Then

$\left(x_{k}\right)=k^{m} \in\left[\hat{c}_{\Delta_{v}^{m}}, F, p, q, u\right]_{l_{\infty}}$ but $\left(\alpha_{k} x_{k}\right) \notin\left[\hat{c}_{\Delta_{v}^{m}}, F, p, q, u\right]_{l_{\infty}}$ when $\alpha_{k}=(-1)^{k}$ for all $k \in \square$. Hence

$\left[\hat{c}_{\Delta_{v}^{m}}, F, p, q, u\right]_{l_{\infty}}$ is not solid. The other cases can be proved on considering similar examples.

Theorem 2.9: The sequence spaces $\left[\hat{c}_{\Delta_{v}^{m}}, F, p, q, u\right]_{z}$ for $z=l_{\infty}, c$ and $c_{0}$, are not symmetric for $m>0$. 
Proof: Let $X=\square, f_{k}(x)=x, p_{k}=1, u_{k}=1, v_{k}=1$ for all $k \in \square$ and $q(x)=|x|$. Then consider the sequence $\left(x_{k}\right)=\left(k^{m}\right)$, then $x \in\left[\hat{c}_{\Delta_{v}^{m}}, F, p, q, u\right]_{z}$ for $z=l_{\infty}, c$ and $c_{0}$.

Let $\left(y_{k}\right)$ be a rearrangement of $\left(x_{k}\right)$, which is defined as follows:

$\left(y_{k}\right)=\left\{x_{1}, x_{2}, x_{4}, x_{3}, x_{9}, x_{5}, x_{16}, x_{6}, x_{25}, x_{7}, x_{36}, x_{8}, x_{14}, x_{10}, \ldots\right\}$.

Then $\left(y_{k}\right) \notin\left[\hat{c}_{\Delta_{v}^{m}}, F, p, q, u\right]_{z}$ for $z=l_{\infty}, c$ and $c_{0}$.

Theorem 2.10: The sequence space $\left[\hat{c}_{\Delta_{v}^{m}}, F, p, q, u\right]_{c_{0}}$ is not a sequence algebra.

Proof: Let $X=\square, f_{k}(x)=x, p_{k}=1, u_{k}=1, v_{k}=1$ for all $k \in \square$ and $q(x)=|x|$. Then consider sequence $x=\left(k^{m-2}\right)$ and $y=\left(k^{m-2}\right)$, then

$x, y \in\left[\hat{c}_{\Delta_{v}^{m}}, F, p, q, u\right]_{c_{0}}$ but $x \cdot y \notin\left[\hat{c}_{\Delta_{v}^{m}}, F, p, q, u\right]_{c_{0}}$.

For other sequences consider $x=\left(k^{m}\right)$ and $y=\left(k^{m}\right)$

Theorem 2.11: The sequence spaces for $z=l_{\infty}, c$ and $c_{0}$ are not convergence free.

Proof: Let $X=\square, f_{k}(x)=x$, for all $x \in[0, \infty), m=1, p_{k}=1, u_{k}=1, v_{k}=1$, for all $k \in \square$. Then $\left(x_{k}\right) \in z(\Delta)$, for $z=l_{\infty}, c$ and $c_{0}$. Consider the sequence $\left(y_{k}\right)$ defined as $\left(y_{k}\right)=k^{2}$ for all $k \in \square$. Then the sequence $\left(y_{k}\right)$ neither belongs to $c_{0}(\Delta)$ nor $c(\Delta)$ nor to $l_{\infty}(\Delta)$. Hence the sequence spaces are not convergence free.

\section{Conclusion}

The concept of difference sequence, however introduced recently, has attracted many researchers because of its broad applications. The topological properties of the newly obtained difference sequence spaces are significant in their own right, on one hand and give rise to many questions regarding the inclusion relation among themselves.

\section{References}

[1]. H. Kizmaz, On certain sequence spaces, Canad. Math Bull, 24 (2), 1981, 169-176.

[2]. M. Et, and R. Çolak, On some generalized difference sequence spaces, Soochow J Math, 21 (4), 1995, 377-386.

[3]. M. Et, and A. Esi, On Köthe-Toeplitz duals of generalized difference sequence spaces, Bull Malaysian Math Sci. Soc, 23 (1), 2000, 25-32.

[4]. C. Asma, , and M. Et, On v-invariant sequence spaces, Soochow J Math, 24 (4), 1998, 305-308.

[5]. C. A. Bektas, M. Et, and R. Colak, Generalized difference sequence spaces and their dual spaces, J Math Anal Appl, 292 (2), 2004, $423-432$.

[6]. R. Colak, On invariant sequence spaces, Erciyes Univ Journal of Sci., 5, 1989, 881 - 888

[7]. M. Isik, On statistical convergence of generalized difference sequences, Soochow J Math, 30 (2), 2004, 197-205.

[8]. M. Mursaleen, Generalized spaces of difference sequences, J Math Anal Appl, 203 (3), 1996, 738-745.

[9]. H. Nakano, Modular sequence spaces, Proc. Japan Acad, 27, 1953, 508-512.

[10]. E. Kolk, Inclusion theorems for some sequence spaces defined by a sequence of moduli, Acta Comment. Univ. Tartu, 970, 1994, :65-72.

[11]. A. K. Gaur and Mursaleen, Difference sequence spaces defined by a sequence of moduli, Demons. Math., 31, 1998, 275 - 298

[12]. V. A. Khan, Some inclusion relations between the difference sequence spaces defined by sequence of moduli, J. Indian Math Soc. $73(1-2), 2006,77-81$.

[13]. I. J. Maddox, Sequence spaces defined by a modulus, Math Proc Camb. Philos. Soc, 100, 1996, 161-166.

[14]. W. H. Ruckle, FK spaces in which the sequence of coordinate vectors is bounded, Canad J Math, 25, 1973, 973-978.

[15]. V. K. Bhardwaj, A generalization of a sequence space of Ruckle, Bull Calcutta Math Soc, 95 (5), 2003, 411-420.

[16]. T. Bilgin, The sequence space $\ell(\mathrm{p}, \mathrm{f}, \mathrm{q}, \mathrm{s})$ on seminormed spaces, Bull Calcutta Math Soc, 86, 1994, $295-304$.

[17]. J. Connor, On strong matrix summability with respect to a modulus and statistical convergence, Canad Math Bull , 32 (2), $1989,194-198$.

[18]. A. Esi, Some new sequence spaces defined by a modulus function, Istanbul “Univ Fen Fak Mat Derg, 55/56, 1996/97, 17-21.

[19]. M. Başarir, On some new sequence spaces and related matrix transformations, Indian J Pure Appl Math, 26 (10), 1995, $1003-1010$.

[20]. I. J. Maddox, Sequence spaces defined by a modulus, Math. Proc. Camb. Phil. Soc., 100, 1986, $161-166$.

[21]. A. Gülcan and Ç.A. Bektas, On some topological properties of generalized difference sequence spaces defined by a sequence of moduli, World Applied Sciences Journal, 12 (6), 2011, 890 - 894.

[22]. G. G. Lorentz, A contribution to the theory of divergent series, Acta Math., 80, 1948, $167-190$

[23]. I. J. Maddox, Spaces of strongly summable sequences, Quart J Math Oxford, 2 (18), 1967, 345-355.

[24]. I. J. Maddox, A new type convergence, Math. Proc. Camb. Phil. Soc., 83, 1978, 61 - 64.

[25]. S. Nanda, Strongly almost convergent sequences, Bull. Cal Math/ Soc., 76, 1984, 236 - 240.

[26]. V. A. Khan and A. Ahmad, On some generalized difference sequence spaces defined by a sequence of moduli, IJRRAS, 7(2), 2011, $106-110$. 